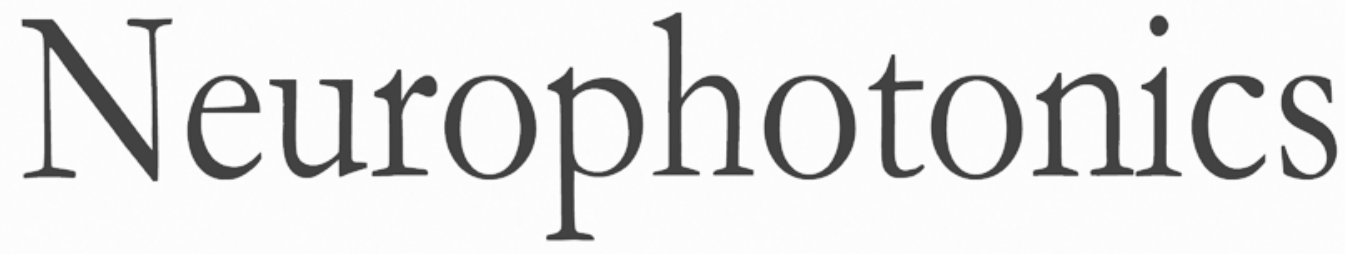

\title{
Fluorescence-guided resection of brain tumor: review of the significance of intraoperative quantification of protoporphyrin IX fluorescence
}

\author{
Zheng Huang \\ Songsheng Shi \\ Haixia Qiu \\ Desheng Li \\ Jian Zou \\ Shaoshan $\mathrm{Hu}$
}




\title{
Fluorescence-guided resection of brain tumor: review of the significance of intraoperative quantification of protoporphyrin IX fluorescence
}

\author{
Zheng Huang, ${ }^{\mathrm{a}, *}$ Songsheng Shi, ${ }^{\mathrm{b}}$ Haixia Qiu, ${ }^{\mathrm{c}}$ Desheng $\mathrm{Li}^{\mathrm{d}}{ }^{\mathrm{d}}$ Jian Zou, ${ }^{\mathrm{a}}$ and Shaoshan $\mathrm{Hu}^{\mathrm{e}}$ \\ ${ }^{a} F u j i a n$ Normal University, MOE Key Laboratory of OptoElectronic Science and Technology for Medicine, 8 Shangsan Road, Fuzhou 350007, China \\ ${ }^{\mathrm{b}}$ Fujian Medical University, Union Hospital, Department of Neurosurgery, 29 Xinquan Road, Fuzhou 350001, China \\ ${ }^{\circ}$ Chinese PLA General Hospital, Department of Laser Medicine, 28 Fuxing Road, Beijing 100039, China \\ dAffiliated Hospital of Academy of Military Medical Sciences, Department of Neurosurgery, 8 Dongda Avenue, Beijing 100071, China \\ ${ }^{\mathrm{e}}$ The Second Affiliated Hospital of Harbin Medical University, Department of Neurosurgery, 246 Xuefu Road, Harbin 150001, China
}

\begin{abstract}
Surgical removal of tumor mass is a common approach in the management of brain tumors. However, the precise delineation of normal tissue from tumor tissue for a complete resection of tumor mass in brain tumor surgery remains a difficult task for neurosurgeons. Aminolevulinic acid (ALA)-mediated exgogenous fluorescence of protoporphyrin IX (PpIX) is a sensitive approach for tumor imaging. Recent studies suggest that the use of ALA/PpIX-mediated fluorescence-guided resection (FGR) or fluorescence-guided surgery can enable more accurate and complete resection of brain tumors, especially when used in quantitative fashion. This review will highlight the current progress in PpIX-mediated FGR and discuss technical challenges in intraoperative quantification of intracellular PpIX fluorescence during FGR of brain tumor. $\odot 2017$ Society of Photo-Optical Instrumentation Engineers (SPIE) [DOI: 10.1117/1.NPh.4.1.011011]
\end{abstract}

Keywords: fluorescence; quantification; aminolevulinic acid; protoporphyrin IX; fluorescence-guided resection; brain tumor. Paper 16042SSVRR received Jul. 1, 2016; accepted for publication Dec. 20, 2016; published online Jan. $12,2017$.

\section{Introduction}

Malignant gliomas constitute $35 \%$ to $45 \%$ of primary brain tumors and their incidence is $\sim 5$ in 100,000. They are classified as WHO grade IV and 5-year survival rate is $<3 \%$. Glioblastomas account for $\sim 60 \%$ to $70 \%$ of malignant gliomas, anaplastic astrocytomas for $10 \%$ to $15 \%$, and anaplastic oligodendrogliomas and anaplastic oligoastrocytomas together for $10 \%$, respectively. ${ }^{1}$ The trend of incidence of these tumors has increased slightly over the past years, especially in the elderly, whereas the trend of 5-year survival has also increased slightly. ${ }^{2}$ High-grade gliomas are rapidly progressive brain tumors and they generally tend to infiltrate adjacent normal brain in a diffuse manner, which poses a big challenge for a clear demarcation of the tumor and is a major factor in therapeutic failure. ${ }^{3}$

Surgical removal of brain tumor mass is the most commonly used approach in the management of brain tumors. ${ }^{1,3}$ The resection strategy might benefit patients through effectively alleviating the mass effect of tumor on neurological tissue, facilitating histopathological diagnosis of the tumor, reducing the risk of recurrence, and helping both physician and patient to determine the strategy of follow-up treatment. Hence, the precise delineation of normal from tumor tissue and extent of resection are of paramount importance in brain tumor surgery and yet these goals remain a difficult task for neurosurgeons. On the one hand, an aggressive and extent resection might enhance the chance of survival and quality of life for the patients. In this regard, the strategy of tumor resection procedures is to maximize

*Address all correspondence to: Zheng Huang, E-mail: zheng_huang@msn .com tumor mass removal and meanwhile to minimize neurological damage. On the other hand, in order to achieve this goal, an accurate identification of tumor margins during craniotomy is critical but this is still technically challenging. ${ }^{4}$

Prior to craniotomy, brain tumor and margin are usually assessed by computerized tomography (CT) and/or magnetic resonance images (MRI). During resection, the margins might be confirmed or determined intraoperatively by neurosurgeons by visual inspection under bright light and information provided by advanced surgical navigation systems including CT, MRI, and intraoperative ultrasound and MRI. However, during the craniotomy, the accuracy of the margin demarcation by the neurosurgeon's visual inspection can be arguable with or without the aid of modern imaging tools. ${ }^{3,4}$ Therefore, there is a clear clinical need for the development of an effective real-time demarcation tool that allows intraoperative in situ detection of tumor margins with high sensitivity and specificity. Over the past few years, several optical spectroscopy-based techniques including fluorescence imaging have been developed and tested to meet this need. ${ }^{5,6}$ This review will highlight the usefulness and technical challenges in fluorescence-guided resection (FGR) or fluorescence-guided surgery (FGS) of brain tumor that utilizes protoporphyrin IX (PpIX).

\section{Protoporphyrin IX Fluorescence}

In general, optical spectroscopic techniques, also known as "optical biopsy," utilize light sources ranging from the ultraviolet (UV) to the infrared, analyze light-tissue interaction and collect information on either reflected light (reflectance spectroscopy) or light interactions with biological tissue (considered as turbid media) or changes in wavelengths (fluorescence and 
<smiles>NCC(=O)CCC(=O)OCl</smiles>

(a)

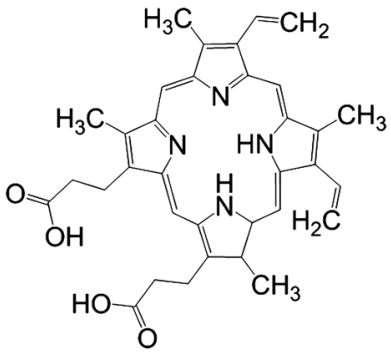

(b)
Fig. 1 Chemical structure of (a) ALA and (b) PpIX.

Raman spectroscopy). Among these light-based techniques, recently, fluorescence-guided tumor resection using native (or endogenous) fluorophores (i.e., autofluorescence) or exgogenous fluorescent probes has demonstrated great potential for identifying tumor margins intraoperatively and for maximizing the resection of glioma. One successful example is the use of $\delta$ or 5-aminolevulinic acid (5-ALA or ALA)-mediated exgogenous fluorescence to facilitate a complete or aggressive resection of brain tumor. ${ }^{3,4,7,8}$ Available clinical data suggest that the ALA-based approach is comparable if not superior to fluorescein fluorescence examination in terms of tumor demarcation and high-degree resection. ${ }^{9}$

$\delta$ - or 5-Aminolevulinic acid hydrochloride [MW 167.59, Fig. 1(a)], a precursor of hemoglobin in the heme biosynthetic pathway, can be used to stimulate porphyrin production in cancer cells.

Numerous clinical studies conducted in Europe and North America demonstrate that the administration of nonfluorescent prodrug ALA can lead to a highly intracellularly selective production and accumulation of PpIX[C34H34N4O4, MW 562.66, Fig. 1(b)] in gliomas, which might be attributed to the deficient blood brain barrier (BBB) in the cancerous areas and an altered activity level of several enzymes in heme metabolism. PpIX is a highly fluorescent compound. When excited with light of appropriate wavelength (e.g., $405 \mathrm{~nm}$ ), PpIX re-emits a distinguished red fluorescence in the visible region with characteristic peaks at $\sim 635$ and $704 \mathrm{~nm}$ in biological sample (Fig. 2). ${ }^{10,11}$

\section{Protoporphyrin IX Mediated Fluorescence- Guided Resection-History and Current Status}

The use of ALA/PpIX to label malignant gliomas for the complete tumor removal was first reported by Stummer et al. In their pilot study of nine patients with malignant gliomas, $10 \mathrm{mg} \mathrm{ALA} /$ $\mathrm{kg}$ body weight was administered orally $3 \mathrm{~h}$ before the induction of general anesthesia. Intraoperatively, red porphyrin fluorescence was observed with a 455 -nm long-pass filter under excitation with the violet-blue band of xenon light (375 to $440 \mathrm{~nm}$ ). A total of 89 fluorescing and nonfluorescing biopsies taken from the tumor perimeters were examined histologically, which demonstrated $85 \%$ sensitivity and $100 \%$ specificity for the detection of malignant tissue. For seven out of nine patients, visible porphyrin fluorescence led to further resection of the tumor. ${ }^{12}$ Their positive outcome led to the development of a fluorescenceguided microsurgical resection system by simply modifying the operating microscope to enable fluorescence-guided tumor removal. A commercially available liquid light guide of $4.8 \mathrm{~mm}$ diameter was coupled to the light inlet of the surgical microscope and used for the fluorescence examination of the patient's tumor cavity. The liquid light guide was manually held during the examination. Further by integrating a 3-chip color CCD camera optimized for red light detection, fluorescence images are able to be recorded. The ALA/PpIX FGR or FGS was used for over 50 patients and no obvious side effects related to the oral administration of ALA at the dosage of $20 \mathrm{mg} / \mathrm{kg}$ were reported. This dose is now used in ALA/PpIX-mediated FGR worldwide. $^{13}$

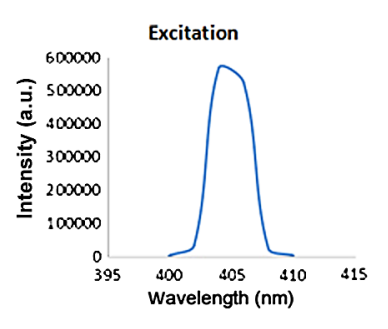

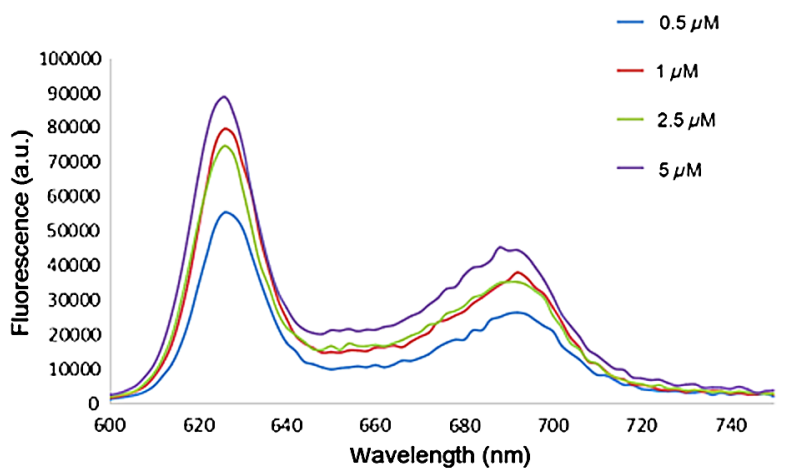

(a)

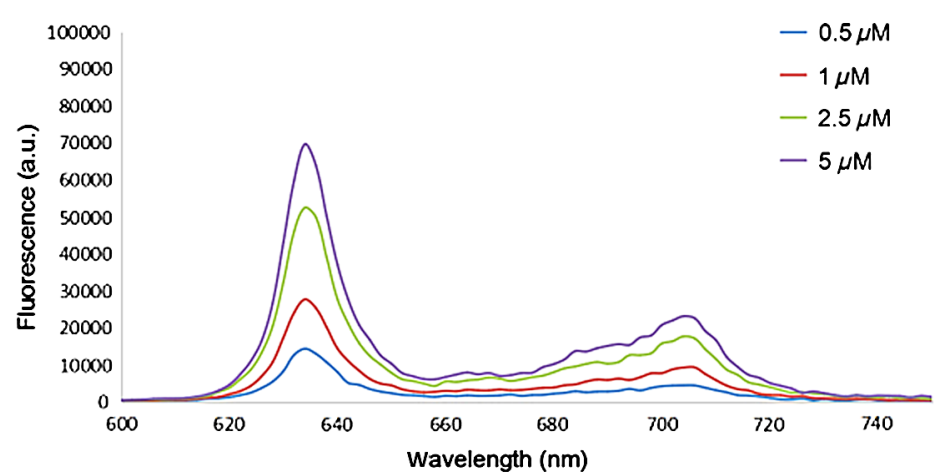

(b)

Fig. 2 Excitation and emission spectra of PpIX. (a) PpIX in DMSO: $\mathrm{H}_{2} \mathrm{O}(1: 3)$ and (b) PpIX in $2 \%$ Intralipid. Insert: bean profile of UV excitation used to obtain the emission spectrum of (a) and (b). The peak wavelength of excitation is $405 \mathrm{~nm}$. 
Eighteen years after its first report, identifying tumor margins with ALA-induced PpIX fluorescence in real time is now effectively used for FGR of high- and low-grade gliomas. Liquid light guide, surgical microscopes or fiber-optic probes can be used for fluorescence detection. FGR leads to a significant increase in the complete resection rate of diffusely growing tumors and to a significant improvement in progression-free survival rate and progression-free survival prolongation as compared to conventional resection under white light. ${ }^{3,4}$

ALA-induced PpIX has been considered as an intraoperative biomarker since ALA-induced intracellular PpIX is a targeting biomarker for a variety of intracranial tumors beyond high grade gliomas. ${ }^{14}$ FGR has also been tested on excised tissue samples during stereotactic biopsy using surgical microscopes, which offers the diagnostic yield of the fluorescent samples of $100 \%$. The fluorescence intensity is correlated to the cell density and the PpIX concentration. The high specificity to malignant tissue and the sensitivity to GBM of ALA-induced PpIX fluorescence indicate that ALA induced PpIX fluorescence is a powerful marker for intraoperative identification of anaplastic foci according to the histopathological WHO criteria in diffusely infiltrating gliomas with nonsignificant contrast-enhancement on MRI. Therefore, application of ALA optimizes tissue sampling for precise histopathological diagnosis independent of brain-shift. ${ }^{15}$

The current goal in resecting malignant gliomas is maximal removal (if possible) of the contrast-enhancing tumor. However, a complete resection of the contrast-enhancing tumor is achieved only in a minority of patients, mainly due to the difficulty in distinguishing the infiltrating tumor from normal adjacent brain tissue during surgery at the tumor margin using conventional surgical microscopy under white-light illumination. ALA/PpIX FGR permits the intraoperative visualization of malignant glioma tissue and enables neurosurgeons to achieve a significantly higher rate of complete resections. Consequently, ALA/PpIX FGR has become an indispensable surgical technique and standard of care at many neurosurgical departments around the world. ${ }^{7,8}$

Although not yet standardized universally, the optical components in ALA/PpIX FGR systems used by neurosurgical departments of different countries are similar. The commonly applied excitation wavelength for diagnostic purposes is blue light (e.g., $405 \mathrm{~nm}$ ) which can be delivered from a coherent light source (e.g., laser) or noncoherent light source (e.g., narrow band LED). A recent study suggests that for achieving deeper tissue penetration the excitation wavelength of $633 \mathrm{~nm}$ seems to be the wavelength of choice for PpIX-assisted detection of high-grade gliomas in stereotactic biopsy. ${ }^{16}$

For ALA/PpIX FGR, ALA, dissolved in orange juice or water, is administered orally in a recommended dose of $20 \mathrm{mg}$ / $\mathrm{kg}$ body weight for brain tumor resection. A recent study indicates that the application of ALA at $5 \mathrm{mg} / \mathrm{kg}$ is equally reliable and effective as the higher dose (i.e., $20 \mathrm{mg} / \mathrm{kg}$ ). In this study, a fluorescence spectroscopy system with a handheld fiber-optic probe was used for performing the quantitative fluorescence measurements. As expected, no PpIX fluorescence was detected in the skin of the patients when using ALA dose of $5 \mathrm{mg} / \mathrm{kg}$, which further reduces the risk of cutaneous phototoxicity. ${ }^{17}$

\section{Clinical Significance of Intraoperative Quantification of PpIX Fluorescence}

To date, the intraoperative observation of PpIX fluorescence in the cavity wall via a modified surgical microscope for tumor delineation has largely been subjective. This approach has a very high specificity but low sensitivity for zones with poor tumoral infiltration. It is limited in its sensitivity for identifying low (but significant) levels of accumulated PpIX in a tumor, potentially leaving some resectable tumor unidentified. ${ }^{18}$ Numerous ex vivo studies support the clinical observation that the PpIX fluorescence signal intensity is correlated to the PpIX concentration and the cancer cell density.

Valdes et al. ${ }^{14,19,20}$ reported their series of study on the quantitative measurement of ALA-induced PpIX concentrations in vivo using a fiber-optic probe and light-transport modeling. Their preliminary results demonstrate the correlation between PpIX concentration in brain tumor tissues and their malignancy profile, which indicates that PpIX fluorescence may be a visual biomarker for tumor-targeting with a diagnostic performance that exceeds subjective visible assessments when measured quantitatively. They further demonstrate that PpIX fluorescence is quantitatively related at the microscopic level to increase malignancy in both low- and high-grade gliomas. The diagnostically significant but visually imperceptible concentrations of PpIX can be quantitatively measured using the fiber-optic approach in vivo and used to delineate normal from tumoral tissue across a range of low- and high-grade gliomahistologies. These works provide a rationale for improving the in vivo quantitative detection of PpIX fluorescence to enable the neurosurgeon to achieve a highly accurate real-time assessment of the tumor margin, overcome shortcomings of the human visual perception of fluorescence, leading to optimal tumor resection.

\section{Challenges in PpIX Fluorescence Quantification}

There has been a long-term interest in the in vivo quantification of fluorescent markers in biological tissue since the accurate quantification of biomarker fluorescence is critical to reveal the functional and/or structural information of disease. However, there are several challenges in in vivo fluorescence quantification since other than the concentration of the fluorescent marker itself the fluorescence measured in vivo depends on many parameters, including the tissue intrinsic autofluorescence, tissue-detector geometry, the sensitivity of optical setup, and the absorbing and scattering properties of the tissue, photobleaching and photoproducts. All can influence the accuracy of fluorescence quantification if not controlled or corrected properly.

In the middle 1980s and 1990s, several quantitative approaches were proposed for the quantification of hematoporphyrin derivative (HPD) through fluorescence measurement in solid tumor after a systemic administration of HPD. ${ }^{21-25}$ Based on the characteristic absorption and emission profile of HPD, the raw uncorrected fluorescence signal can be corrected through the calculation of the ratio of the administrated marker signal, i.e., red fluorescence over the reference signal, i.e., autofluorescence and/or green fluorescence. The fluorescence signals of interest can be obtained from single or multiple emission wavelengths or wave bands under a single or double wavelength or wave band excitation, which determines the setup of the fluorescence detection system and vise verse. Bogaards et al. evaluated the performance of these representative methods by varying input parameters of irradiance, excitation geometry, collection efficiency, autofluorescence background, melanin content, blood volume, blood oxygenation, and tissue scattering using optical properties representing those for human skin. They noticed 
that the uncertainty in the fluorescence signal is affected by probe geometry, i.e., whether the site of interest can be relocated before and after fluorescent marker administration. ${ }^{26}$

The clinical potentials of ALA/PpIX FGR in glioma treatment have brought up a renewed interest in ex vivo and in vivo quantification of PpIX in brain tissue. In light of the similarity in the absorption and emission profile between the mixture form of HPD and the monomer form of PpIX, the in vivo fluorescence signal of PpIX can also be quantified from single or multiple emission wavelengths under a single source or double source excitation. Ishihara et al. used the fluorescence intensity ratio, i.e., the ratio of the peak emission intensity to reflected excitation light intensity after subtracting the extrapolated autofluorescence curve, to quantify the PpIX fluorescence intensity in diffusely infiltrating astrocytomas. ${ }^{27}$ Technically, it is possible to extract more information from the fluorescent signal with advanced optical detection methods. For instance, as demonstrated in recent clinical studies, ${ }^{28,29}$ with the use of a handheld fiber-optic probe that can be placed directly on the cavity wall, the spectroscopic method has been developed to first interrogate the same tissue with white light to collect the remitted white light reflectance or diffuse reflectance spectra and to compute the wavelength-dependent absorption and scattering values of the tissue, and then interrogate with violet-blue light to collect the spectrally resolved fluorescence emissions. Using the obtained values (i.e., optical properties and fluorescence) to determine an absorption- and scattering-independent value for the fluorescence and finally fitting the known spectra of the major fluorophores in the tissue, one can derive the absolute concentration of PpIX.

The configuration of handheld fiber-optic probe needs to be optimized for sensitively and simultaneously collecting the tissue fluorescence and diffuse reflectance spectra in vivo. A proper light-transport modeling and matching correction algorithm needs to be validated for real-time quantitative measurement. ${ }^{30}$ In the handheld detection mode, correction of motion artifacts might improve the bed-side real-time measurement. ${ }^{31}$

Fluorescence molecular tomography has been used as a means of quantitatively imaging fluorescent molecular probes in three dimensions in living systems. Although autofluorescence and green fluorescence are often used as the internal reference in ALA/PpIX FGR, at the cellular level, since some biosensors, e.g., the constitutive expression of red fluorescence proteins or yellow fluorescence proteins (YFP), are strongly correlated with cell number, the PpIX/YFP fluorescence ratio can be used for the assessment of PpIX accumulation in tissue. ${ }^{32,33}$

\section{Summary}

In summary, available clinical data strongly suggest that the use of ALA/PpIX FGR can enable more accurate and complete resection of brain tumors, especially when used in a quantitative fashion. Quantifying PpIX fluorescence can be achieved from the in vivo simultaneous detection of tissue fluorescence and diffuse reflectance spectra using commercially available handheld fiber-optic probes. PpIX concentration can be obtained after decoupling the specific PpIX fluorescence from other fluorescent signals (e.g., autofluorescence and fluorescent photoproducts) after the consideration of the effect of tissue optical property. The clinical potentials of FGR deserve thoughtful investigation.

\section{Disclosures}

The authors have no conflicts of interest to declare.

\section{Acknowledgments}

This work was funded by the National Natural Science Foundation of China (NSFC, Grant Number 81471703) and Innovation Team Development Project (No. IRT_15R10).

\section{References}

1. T. Mesti and J. Ocvirk, "Malignant gliomas: old and new systemic treatment approaches," Radiol. Oncol. 50(2), 129-138 (2016).

2. R. L. Siegel, K. D. Miller, and A. Jemal, "Cancer statistics," CA: Cancer J. Clin. 65(1), 5-29 (2015).

3. B. J. Quirk et al., "Photodynamic therapy (PDT) for malignant brain tumors-where do we stand," Photodiagn. Photodyn. Ther. 12(3), 530-544 (2015)

4. M. C. Tetard et al., "Experimental use of photodynamic therapy in high grade gliomas: a review focused on 5-aminolevulinic acid," Photodiagn. Photodyn. Ther. 11(3), 319-330 (2014).

5. M. Bahreini, "Role of optical spectroscopic methods in neurooncological sciences," J. Lasers Med. Sci. 6(2), 51-61 (2015).

6. P. A. Valdés et al., "Optical technologies for intraoperative neurosurgical guidance," Neurosurg. Focus 40(3), E8 (2016).

7. C. G. Hadjipanayis, G. Widhalm, and W. Stummer, "What is the surgical benefit of utilizing 5-aminolevulinic acid for fluorescence-guided surgery of malignant gliomas," Neurosurgery 77(5), 663-673 (2015).

8. J. Guyotat et al., "5-Aminolevulinic acid-protoporphyrin IX fluorescence-guided surgery of high-grade gliomas: a systematic review," Adv. Tech. Stand. Neurosurg. 43, 61-90 (2016).

9. F. Acerbi et al., "Fluorescein-guided surgery for malignant gliomas: a review," Neurosurg. Rev. 37(4), 547-557 (2014).

10. M. J. Colditz and R. L. Jeffree, "Aminolevulinic acid (ALA)-protoporphyrin IX fluorescence guided tumour resection. Part 1: clinical, radiological and pathological studies," J. Clin. Neurosci. 19(11), 1471-1474 (2012).

11. M. J. Colditz, K. V. Leyen, and R. L. Jeffree, "Aminolevulinic acid (ALA)-protoporphyrin IX fluorescence guided tumour resection. Part 2: theoretical, biochemical and practical aspects," J. Clin. Neurosci. 19(12), 1611-1616 (2012).

12. W. Stummer et al., "Intraoperative detection of malignant gliomas by 5aminolevulinic acid-induced porphyrin fluorescence," Neurosurgery 42(3), 518-526 (1998).

13. W. Stummer et al., "Technical principles for protoporphyrin-IX-fluorescence guided microsurgical resection of malignant glioma tissue," Acta Neurochir. 140(10), 995-1000 (1998).

14. P. A. Valdés et al., "Quantitative fluorescence in intracranial tumor: implications for ALA-induced PpIX as an intraoperative biomarker," J. Neurosurg. 115(1), 11-17 (2011).

15. G. Widhalm et al., "5-Aminolevulinic acid induced fluorescence is a powerful intraoperative marker for precise histopathological grading of gliomas with non-significant contrast-enhancement," PLoS One 8(10), e76988 (2013).

16. N. A. Markwardt et al., " $405 \mathrm{~nm}$ versus $633 \mathrm{~nm}$ for protoporphyrin IX excitation in fluorescence-guided stereotactic biopsy of brain tumors," J. Biophotonics 9(9), 901-912 (2016).

17. N. Haj-Hosseini et al., "Low dose 5-aminolevulinic acid: implications in spectroscopic measurements during brain tumor surgery," Photodiagn. Photodyn. Ther. 12(2), 209-214 (2015).

18. A. Johansson et al., "5-Aminolevulinic acid-induced protoporphyrin IX levels in tissue of human malignant brain tumors," Photochem. Photobiol. 86(6), 1373-1378 (2010).

19. P. A. Valdés et al., " $\delta$-aminolevulinic acid-induced protoporphyrin IX concentration correlates with histopathologic markers of malignancy in human gliomas: the need for quantitative fluorescence-guided resection to identify regions of increasing malignancy," Neuro-Oncol. 13(8), 846-856 (2011).

20. P. A. Valdés et al., "Combined fluorescence and reflectance spectroscopy for in vivo quantification of cancer biomarkers in low- and high-grade glioma surgery," J. Biomed. Opt. 16(11), 116007 (2011).

21. A. Profio, "Laser excited fluorescence of hematoporhyrinderivative for diagnosis of cancer," IEEE J. Quantum Electron. 20, 1502-1507 (1984).

22. S. Montan, K. Svanberg, and S. Svanberg, "Multicolor imaging an contrast enhancement in cancer-tumor localization using laser induced 
fluorescence in hematoporphyrin-derivative-bearing tissue," Opt. Lett. 10, 56-58 (1985).

23. R. Baumgartner et al., "A fluorescence imaging device for endoscopic detection of early stage cancer-instrumental and experimental studies," Photochem. Photobiol. 46, 759-763 (1987).

24. M. Sinaasappel and H. J. C. M. Sterenborg, "Quantification of the hematoporphyrin derivative by fluorescence measurement using dualwavelength excitation and dual-wavelength detection," Appl. Opt. 32, 541-548 (1993).

25. A. E. Saarnak et al., "Influence of tumour depth, blood absorption and autofluorescence on measurement of exogenous fluorophores in tissue," Laser Med. Sci. 13, 22-31 (1998).

26. A. Bogaards, H. J. Sterenborg, and B. C. Wilson, "In vivo quantification of fluorescent molecular markers in real-time: a review to evaluate the performance of five existing methods," Photodiagn. Photodyn. Ther. 4(3), 170-178 (2007).

27. R. Ishihara et al., "Quantitative spectroscopic analysis of 5-aminolevulinic acid-induced protoporphyrin IX fluorescence intensity in diffusely infiltrating astrocytomas," Neurol. Med. Chir. 47(2), 53-57 (2007).

28. R. Weersink et al., "Noninvasive measurement of fluorophore concentration in turbid media with a simple fluorescence/reflectance ratio technique," Appl. Opt. 40(34), 6389-6395 (2001).

29. A. Kim et al., "Quantification of in vivo fluorescence decoupled from the effects of tissue optical properties using fiber-optic spectroscopy measurements," J. Biomed. Opt. 15(6), 067006 (2010).
30. S. A. de Visscher et al., "In vivo quantification of photosensitizer concentration usingfluorescence differential path-length spectroscopy: influence of photosensitizer formulation and tissue location," $J$. Biomed. Opt. 17(6), 067001 (2012).

31. J. W. Barker et al., "Correction of motion artifacts and serial correlations for real-time functional near-infrared spectroscopy," Neurophotonics 3(3), 031410 (2016)

32. J. E. Lawrence et al., "Quantification of protoporphyrin IX accumulation in glioblastoma cells: a newtechnique," ISRN Surg. 4, 405360 (2014).

33. Y. Shen, T. Lai, and E. R. Campbell, "Red fluorescent proteins (RFPs) and RFP-based biosensors for neuronal imaging applications," Neurophotonics 2(3), 031203 (2015).

Zheng Huang received his MD from Suzhou Medical College and $\mathrm{PhD}$ in life science from King's College of London University. He is currently a professor at the Medical Photonics Center and the College of Photonic and Electronic Engineering of the Fujian Normal University. He has been actively involved in basic and clinical research of photodynamic therapy in the USA since 2000 . His research interests include photomedicine, biomedical optics, and biomedical imaging. He has published over 125 peer-reviewed articles and 5 book chapters. He has been a SPIE member since 2001.

Biographies for the other authors are not available. 